\title{
Article
}

\section{Weak implicative UP-filters of UP-algebras}

\author{
Daniel A. Romano ${ }^{1, *}$ and Young Bae Jun ${ }^{2}$ \\ 1 International Mathematical Virtual Institute 6, Kordunaška Street, 78000 Banja Luka, Bosnia and Herzegovina. \\ 2 Department of Mathematics Education, Gyeongsang National University, Jinju 52828, Korea. \\ * Correspondence: bato49@hotmail.com
}

Received: 6 November 2020; Accepted: 30 November 2020; Published: 2 December 2020.

Abstract: The concept of weak implicative UP-filters in UP-algebras is introduced and analyzed. Some characterizations of weak implicative UP-filters are derived with the using of some other filter types in such algebras.

Keywords: UP-algebra, UP-filter, implicative UP-filter, comparative UP-filter, $x$-allied UP-filter, weak implicative UP-filter.

MSC: 03G25, 06F35.

\section{Introduction}

$\mathbf{P}$ rabpayak and Leerawat [1,2] introduced the notion of a KU-algebra. In [3], Iampan introduced a new algebraic structure, called a UP-algebra, which is a generalization of a KU-algebra. He studied a UP-subalgebra and a UP-ideal. Somjanta et al. [4] introduced the concept of UP-filters. Then Jun and Iampan ([5-7]) developed several types of filters in these algebras such as comparative and implicative UP-filters. Romano also took part in analyzing filter properties in UP-algebras (for example [8,9]).

In this paper, we introduce the notion of a weak implicative UP-filter which is located between UP-filter and implicative UP-filter, and further studies the relationship between various types of UP-filters, that is, relations between UP-filter, implicative UP-filter, weak implicative UP-filter, comparative UP-filter and allied UP-filter. We provide conditions for a weak implicative UP-filter to be an implicative UP-filter. We consider conditions for a UP-filter to be a weak implicative UP-filter. We suggest the conditions for a weak implicative UP-filter to be an allied UP-filter.

\section{Preliminaries}

An algebra $A=(A, \cdot 0)$ of type $(2,0)$ is called a UP-algebra (see [3]) if it satisfies the following axioms:

(UP-1) $(\forall x, y, \in A)((y \cdot z) \cdot((x \cdot y) \cdot(x \cdot z))=0)$,

(UP-2) $(\forall x \in A)(0 \cdot x=x)$,

(UP-3) $(\forall x \in A)(x \cdot 0=0)$,

(UP-4) $(\forall x, y \in A)((x \cdot y=0 \wedge y \cdot x=0) \Longrightarrow x=y)$.

In this algebra, the order relation ' $\leqslant$ ' is defined as follows

$$
(\forall x, y \in A)(x \leqslant y \Longleftrightarrow x \cdot y=0) .
$$

A subset $F$ of a UP-algebra $A$ is called a UP-filter of $A$ (see [4]) if it satisfies the following conditions:

(F-1) $0 \in F$,

(F-2) $(\forall x, y \in A)((x \in F \wedge x \cdot y \in F) \Longrightarrow y \in F)$.

It is clear that every UP-filter $F$ of a UP-algebra $A$ satisfies:

(1) $(\forall x, y \in A)((x \in F \wedge x \leqslant y) \Longrightarrow y \in F)$.

The family $\mathfrak{F}(A)$ of all UP-filters of a UP-algebra $A$ is not empty and forms a complete lattice. 
Definition 1. ([5], Definition 1) A subset $F$ of a UP-algebra $A$ is called an implicative UP-filter of $A$ if it satisfies the following conditions:

(F-1) $0 \in F$ and

(IF) $(\forall x, y, \in A)((x \cdot(y \cdot z) \in F \wedge x \cdot y \in F) \Longrightarrow x \cdot z \in F)$.

Example 1. Let $S=\{1,2,3,0\}$ and operations '.' defined on $A$ as follows:

\begin{tabular}{l|llll}
$\cdot$ & 1 & 2 & 3 & 0 \\
\hline 1 & 0 & 0 & 0 & 0 \\
2 & 1 & 0 & 3 & 0 \\
3 & 1 & 2 & 0 & 0 \\
0 & 0 & 1 & 2 & 3
\end{tabular}

Then $A=(A, \cdot, 0)$ is a UP-algebra where the order relation ' $\leqslant$ ' is defined as follows $\leqslant=$ $\{(0,0),(1,1),(2,2),(3,3),(1,0),(2,0),(3,0),(1,2),(1,3)\}$. The subsets $\{0\},\{0,2\},\{0,3\}$ and $\{0,2,3\}$ are implicative UP-filters of $A$.

Note that if we put $x=1, y=x$ and $z=y$ in (IF) and use (UP-2), then every implicative UP-filter is a UP-filter (see also [5], Theorem 1). But a UP-filter may not be an implicative UP-filter as seen in the following example.

Example 2. ([5], Example 2) Consider a UP-algebra $A=\{0,1,2,3\}$ with the binary operation '.' given in the following table

\begin{tabular}{l|llll}
$\cdot$ & 0 & 1 & 2 & 3 \\
\hline 0 & 0 & 1 & 2 & 3 \\
1 & 0 & 0 & 1 & 2 \\
2 & 0 & 0 & 0 & 2 \\
3 & 0 & 0 & 0 & 0
\end{tabular}

Here the order relation ' $\leqslant$ ' is given by

$$
\leqslant=\{(0,0),(1,1),(2,2),(3,3),(1,0),(2,0),(2,1),(3,0),(3,1),(3,2)\} .
$$

Then the subset $\{0\}$ is a UP-filter of $A$, but it is not an implicative UP-filter since $2 \cdot(2 \cdot 3)=0 \in\{0\}$ and $2 \cdot 2=0 \in\{0\}$, but $2 \cdot 3=2 \notin\{0\}$.

\section{Weak implicative UP-filters of UP-algebras}

This section introduces weak implicative UP-filter located between UP-filter and implicative UP-filter, and further studies the relationship between various types of UP-filters.

In what follows, let $A$ denote a UP-algebra unless otherwise specified.

Definition 2. A subset $F$ of $A$ is called a weak implicative UP-filter of $A$ if it satisfies the following conditions:

(F-1) $0 \in F$,

(WIF) $(\forall x, y, z \in A)((x \cdot(y \cdot z) \in F \wedge x \cdot y \in F) \Longrightarrow x \cdot(x \cdot z) \in F)$.

Example 3. Let $A=\{0,1,2,3,4\}$ be a set with the operation ' $'$ ' given by

\begin{tabular}{c|ccccc}
$\cdot$ & 0 & 1 & 2 & 3 & 4 \\
\hline 0 & 0 & 1 & 2 & 3 & 4 \\
1 & 0 & 0 & 0 & 0 & 0 \\
2 & 0 & 2 & 0 & 0 & 0 \\
3 & 0 & 2 & 2 & 0 & 0 \\
4 & 0 & 2 & 2 & 4 & 0
\end{tabular}

Then $A$ is a UP-algebra (see Example 1.12 in [3]), and $F:=\{0,2\}$ is a weak implicative UP-filter of $A$. 
Proposition 1. Every weak implicative UP-filter $F$ of A satisfies the following assertion. $(\forall x, z \in A)(x \cdot z \in F \Longrightarrow$ $x \cdot(x \cdot z) \in F)$.

Proof. If we put $y=0$ in (WIF), we immediately get the required implication.

Theorem 1. Every weak implicative UP-filter is a UP-filter.

Proof. If we put $x=0, y=x$ and $z=y$ in (WIF), we get (F-2) by (UP-2).

The following example shows that the converse of Theorem 1 is not true.

Example 4. Let $A=\{0,1,2,3\}$ be a set with the binary operation ' $\cdot$ ' which is given by the following table.

\begin{tabular}{c|cccc}
$\cdot$ & 0 & 1 & 2 & 3 \\
\hline 0 & 0 & 1 & 2 & 3 \\
1 & 0 & 0 & 1 & 1 \\
2 & 0 & 0 & 0 & 1 \\
3 & 0 & 0 & 0 & 0
\end{tabular}

Then $A$ is a UP-algebra (see Example 3 in [6]). The subset $F:=\{0,1,2\}$ is a UP-filter of $A$, but it is not a weak implicative UP-filter of $A$ because $0 \cdot(2 \cdot 3)=1 \in F$ and $0 \cdot 2=2 \in F$ but $0 \cdot(0 \cdot 3)=3 \notin F$.

Lemma 1 ([3]). Every UP-algebra A satisfies the following condition

$$
(\forall x, y \in A)(x \leq y \cdot x) .
$$

Theorem 2. Every implicative UP-filter is a weak implicative UP-filter.

Proof. Let $F$ be an implicative UP-filter of $A$. Then $F$ is a UP-filter of $A$ by Theorem 1 . Let $x, y, z \in A$ be such that $x \cdot(y \cdot z) \in F$ and $x \cdot y \in F$. Then $x \cdot z \in F$ by (IF). Since $x \cdot z \leqslant x \cdot(x \cdot z)$ by Lemma 1, it follows from (1) that $x \cdot(x \cdot z) \in F$. Therefore $F$ is a weak implicative UP-filter of $A$.

The converse of Theorem 2 is not true as seen in the following example.

Example 5. Consider the UP-algebra $A$ which is given in Example 2. The subset $\{0\}$ is a weak implicative UP-filter of $A$ but it is not an implicative UP-filter of $A$.

We provide conditions for a weak implicative UP-filter to be an implicative UP-filter, and hence the following theorem is a characterization of an implicative UP-filter.

Theorem 3. Let $F$ be a weak implicative UP-filter of $A$. Then $F$ is an implicative UP-filter of $A$ if and only if

(2) $(\forall x, y \in A)(x \cdot(x \cdot y) \in F \Longleftrightarrow x \cdot y \in F)$.

Proof. Let $F$ be a weak implicative UP-filter of $A$. Then $F$ be a UP-filter of $A$ by Theorem 1 . Assume that $F$ is an implicative UP-filter of $A$, and let $x, y \in A$ be such that $x \cdot(x \cdot y) \in F$. Since $x \cdot x=0 \in F$, it follows from (IF) that $x \cdot y \in F$. Now suppose $x \cdot y \in F$ for all $x, y \in A$. Since $x \cdot y \leqslant x \cdot(x \cdot y)$ by Lemma 1 , it follows that $x \cdot(x \cdot y) \in F$ by (1). Hence (2) is valid.

Conversely, assume that $F$ satisfies the condition (2). Let $x, y, z \in A$ be such that $x \cdot(y \cdot z) \in F$ and $x \cdot y \in F$. Since $F$ is a weak implicative UP-filter, we get $x \cdot(x \cdot z) \in F$ which implies from (2) that $x \cdot z \in F$. So, $F$ is an implicative UP-filter of $A$.

In the next theorem, we consider conditions for a UP-filter to be a weak implicative UP-filter.

Theorem 4. If a UP-filter $F$ of $A$ satisfies the following condition

(3) $(\forall x, y, z \in A)(x \cdot(y \cdot z) \in F \Longrightarrow(x \cdot y) \cdot(x \cdot(x \cdot z)) \in F)$,

then $F$ is a weak implicative UP-filter of $A$. 
Proof. Let $F$ be a UP-filter of $A$ satisfying the condition (3). Let $x, y, z \in A$ be such that $x \cdot(y \cdot z) \in F$ and $x \cdot y \in F$. Then $(x \cdot y) \cdot(x \cdot(x \cdot z)) \in F$ by (3), and thus $x \cdot(x \cdot z) \in F$ by (F-2). Therefore, $F$ is a weak implicative UP-filter of $A$.

In [5], Jun and Iampan introduced the notion of allied UP-filters in UP-algebras. The concept of allied UP-filters with respect to the element $x$ in $A$ is given by the following definition.

Definition 3. ([6], Definition 4) Let $x$ be a fixed element of $A$. A subset $F$ of $A$ is called an allied UP-filter of $A$ with respect to $x$ (briefly, $x$-allied UP-filter of $A$ ) if it satisfies the conditions

(F-1) $0 \in F$ and

(FA) $(\forall y, z \in A)((x \cdot(y \cdot z) \in F \wedge x \cdot y \in F) \Longrightarrow z \in F)$.

We discuss relationship between allied UP-filters and (weak) implicative UP-filters. The following example shows that any weak implicative UP-filter may not be an $x$-allied UP-filter for some $x \in A$.

Example 6. Consider the UP-algebra in Example 2. It can be proved without major difficulties that the set $F:=\{0,1,2\}$ is a weak implicative UP-filter of $A$ but it is not a 1-allied UP-filter because for $x=1, y=0$ and $z=3$ we have $1 \cdot(0 \cdot 3)=1 \cdot 3=2 \in F$ and $1 \cdot 0=0 \in F$ but $3 \notin F$.

Given an element $x$ of $A$, we suggest the conditions for a weak implicative UP-filter to be an $x$-allied UP-filter.

Theorem 5. Given an element $x$ of $A$, if a weak implicative UP-filter $F$ of $A$ satisfies the following condition

(4) $(\forall y \in A)(x \cdot(x \cdot y) \in F \Longrightarrow y \in F)$,

then $F$ is an $x$-allied UP-filter of $A$.

Proof. Let $F$ be a weak implicative UP-filter of $A$ satisfying the condition (4) for $x \in A$. Let $y, z \in A$ be such that $x \cdot(y \cdot z) \in F$ and $x \cdot y \in F$. Then $x \cdot(x \cdot z) \in F$ by (WIF). It follows from (4) that $z \in F$. Therefore, $F$ is an $x$-allied UP-filter of $A$.

The following example shows that any implicative UP-filter may not be an $x$-allied UP-filter for some $x \in A$.

Example 7. Let $A=\{0, a, b, c\}$ be a set with the binary operation '.' which is given by the following table.

\begin{tabular}{c|cccc}
$\cdot$ & 0 & $\mathrm{a}$ & $\mathrm{b}$ & $\mathrm{c}$ \\
\hline 0 & 0 & $\mathrm{a}$ & $\mathrm{b}$ & $\mathrm{c}$ \\
$\mathrm{a}$ & 0 & 0 & 0 & 0 \\
$\mathrm{~b}$ & 0 & $\mathrm{a}$ & 0 & $\mathrm{c}$ \\
$\mathrm{c}$ & 0 & $\mathrm{a}$ & $\mathrm{b}$ & 0
\end{tabular}

Then $A$ is a UP-algebra (see Example 1.6 in [3]). The subset $F:=\{0, b\}$ is an implicative UP-filter of $A$ (see Example 1 in [5]), but it is not a $c$-allied UP-filter of $A$ because for $x=c, y=0$ and $z=c$ we have $c \cdot(0 \cdot c)=0 \in F$ and $c \cdot 0=0 \in F$ but $c \notin F$.

Corollary 1. Given an element $x$ of $A$, if an implicative UP-filter $F$ of $A$ satisfies the condition (4), then $F$ is an $x$-allied UP-filter of $A$.

Proposition 2. Given an element $x$ of $A$, every $x$-allied UP-filter $F$ of $A$ satisfies the condition (4).

Proof. Let $y \in A$ be such $x \cdot(x \cdot y) \in F$. Since $x \cdot x=0 \in F$, it follows from (FA) that $y \in F$. This completes the proof.

We consider conditions for a UP-filter to be a weak implicative UP-filter.

Theorem 6. Let A be a UP-algebra which satisfies the condition 
(5) $(\forall x, y, z \in A)(x \cdot(y \cdot z)=y \cdot(x \cdot z))$.

If any UP-filter $F$ of $A$ satisfies the following assertion

(6) $(\forall x, y \in A)(x \cdot(x \cdot y)=x \cdot y)$,

then $F$ is a weak implicative UP-filter of $A$.

Proof. It is straightforward by combining Theorem 5 in [5] and Theorem 2.

Definition 4. ([6], Definition 2) A subset $F$ of a UP-algebra $A$ is called a comparative UP-filter of $A$ if it satisfies the following conditions

(F-1) $0 \in F$,

(CF) $(\forall x, y, z \in A)((x \cdot((y \cdot z) \cdot y) \in F \wedge x \in F) \Longrightarrow y \in F)$.

Remark 1. Let us note that condition (CF) is equivalent to the condition

$(\mathrm{CF}(5))(\forall x, y, z \in A)(((y \cdot z) \cdot(x \cdot y) \in F \wedge x \in F) \Longrightarrow y \in F)$

if the UP-algebra $A$ satisfies the condition (5). It should also be noted that if the UP-algebra satisfies condition (5), then condition (3) can be replaced by condition

(3(5)) $(\forall x, y, z \in A)(x \cdot(y \cdot z) \in F \Longrightarrow x \cdot((x \cdot y) \cdot(x \cdot z)) \in F)$.

It is known that any comparative UP-filter is a UP-filter and not viceversa (see Theorem 1 and Example 2 in [6]). We now consider relations between a weak implicative UP-filter and a comparative UP-filter. In the following example, we show that any weak implicative UP-filter may not be a comparative UP-filter.

Example 8. Let $A=\{0,1,2,3\}$ be the UP-algebra which is given in Example 2. The subset $\{0\}$ is a weak implicative UP-filter of $A$ but it is not a comparative UP-filter of $A$ since for $x=0, y=1$ and $z=2$ we have $0 \cdot((1 \cdot 2) \cdot 1)=0 \cdot(1 \cdot 1)=0 \cdot 0=0 \in\{0\}$ and $0 \in\{0\}$ but $1 \notin\{0\}$.

The following example shows that a comparative UP-filter is not a weak implicative UP filter.

Example 9. Let $A=\{0,1,2,3\}$ be the UP-algebra which is given in Example 2. Then the set $F:=\{0,1,2\}$ is a comparative UP-filter of $A$ (see Example 1 in [6]). But it is not a weak implicative UP-filter since $0 \cdot(1 \cdot 3)=$ $2 \in F$ and $0 \cdot 1=1 \in F$ but $0 \cdot(0 \cdot 3)=3 \notin F$.

A UP-algebra $A$ is said to be meet-commutative ([6], Definition 3) if it satisfies the condition

(7) $(\forall x, y \in A)((x \cdot y) \cdot y=(y \cdot x) \cdot x)$.

We provide conditions for a comparative UP-filter to be a weak implicative UP-filter.

Theorem 7. Let A be a meet-commutative UP-algebra which satisfies the condition (5). Then every comparative UP-filter is a weak implicative UP-filter.

Proof. It is straightforward by combining Theorem 3 in [6] and Theorem 2.

We end this section with the following theorem.

Theorem 8. The family $\mathfrak{F}_{w i}(A)$ of all weak implicative UP-filters of $A$ forms a complete lattice and $\mathfrak{F}_{w i}(A) \subseteq \mathfrak{F}(A)$.

Proof. Let $\left\{F_{k}\right\}_{k \in \Lambda}$ be a family of weak implicative UP-filters of $A$ where $\Lambda$ is index set. It is clear that $0 \in$ $\bigcap_{k \in \Lambda} F_{k}$. Let $x, y, z \in A$ be such that $x \cdot(y \cdot z) \in \bigcap_{k \in \Lambda} F_{k}$ and $x \cdot y \in \bigcap_{k \in \Lambda} F_{k}$. Then $x \cdot(y \cdot z) \in F_{k}$ and $x \cdot y \in F_{k}$ for any $k \in \Lambda$. Thus $x \cdot(x \cdot z) \in F_{k}$ for all $k \in \Lambda$. Hence $x \cdot(x \cdot z) \in \bigcap_{k \in \Lambda} F_{k}$. So, the intersection $\bigcap_{k \in \Lambda} F_{k}$ satisfies the condition (WIF). Therefore $\bigcap_{k \in \Lambda} F_{k}$ is a weak implicative UP-filter of $A$. Let $\mathfrak{X}$ be the family of all weak implicative UP-filters containing the union $\bigcup_{k \in \Lambda} F_{k}$. Then $\cap \mathfrak{X}$ is a weak implicative UP-filter of $A$ according to the first part of this proof. If we put $\Pi_{k \in \Lambda} F_{k}=\bigcap_{k \in \Lambda} F_{k}$ and $\sqcup_{k \in \Lambda} F_{k}=\cap \mathfrak{X}$, then $\left(\mathfrak{F}_{w i}(A), \sqcap, \sqcup\right)$ is a complete lattice. 
Corollary 2. Let $X$ be a subset of $A$. Then there exists the unique minimal weak implicative UP-filter of $A$ containing $X$.

Proof. Let $\mathfrak{X}$ be the family of all weak implicative UP-filters of $A$ containing $X$. It is clear that $\cap \mathfrak{X}$ is a unique minimal weak implicative UP-filter containing $X$ according to the second part of the proof of the previous theorem.

Corollary 3. Let a be an arbitrary element of $A$. Then there is the unique minimal weak implicative UP-filter containing a.

Proof. This follows directly from the proof of the previous Corollary if we take $X=\{a\}$.

As we finish this paper, we ask the next question.

Question 1 (Extension property for weak implicative UP-filter). What are the necessary conditions for a UP-filter including a weak implicative UP-filter to be a weak implicative UP-filter?

\section{Conclusion}

The aim of this paper was to study the concept of weak implicative UP-filters of a UP-algebra. Additionally, some links have been established between this type of UP-filter of UP-algebras with other types of filters in these algebras such as implicative, comparative and allied filters. This work can be the basis for further and deeper research of the properties of UP-algebras.

Author Contributions: All authors contributed equally to the writing of this paper. All authors read and approved the final manuscript.

Conflicts of Interest: “The authors declare no conflict of interest."

\section{References}

[1] Prabpayak, C., \& Leerawat, U. (2009). On ideals and congruences in KU-algebras. Scienctia Magna Journal, 5(1), 54-57.

[2] Prabpayak, C., \& Leerawat, U. (2009). On isomorphisms of KU-algebras. Scientia Magna Journal, 5(3), 25-31.

[3] Iampan, A. (2017). A new branch of the logical algebra: UP-algebras. Journal of Algebra and Related Topics, 5(1), 35-54.

[4] Somjanta, J., Thuekaew, N., Kumpeangkeaw, P., \& Iampan, A. (2016). Fuzzy sets in UP-algebras. Annals of Fuzzy Mathematics and Informatics, 12(6), 739-756.

[5] Jun, Y. B., \& Iampan, A. (2019). Implicative UP-filters. Afrika Matematika, 30(7-8), 1093-1101.

[6] Jun, Y. B., \& Iampan, A. (2019). Comparative and Allied UP-Filters. Lobachevskii Journal of Mathematics, 40(1), 60-66.

[7] Jun, Y. B., \& Iampan, A. (2019). Shift UP-filters and decompositions of UP-filters in UP-Algebras. Missouri Journal of Mathematical Sciences, 31(1), 36-45.

[8] Romano, D. A. (2018). Proper UP-filters in UP-algebra. Universal Journal of Mathematics and Applications, 1(2), 98-100.

[9] Romano, D. A. (2018). Some properties of proper UP-filters of UP-algebras. Fundamental Journal of Mathematics and Applications, 1(2), 109-111. 